\title{
Chest tube removal algorithm is associated with decreased chest tube duration in pediatric cardiac surgical patients
}

\author{
Rebecca A. Bertrandt, MD, ${ }^{\mathrm{a}}$ David M. Saudek, MD, ${ }^{\mathrm{b}}$ John P. Scott, MD, ${ }^{\mathrm{c}}$ Michael Madrzak, PA-C, MPAS, ${ }^{\mathrm{d}}$ \\ Mary Beth Miranda, MS, RN, CLSSBB, ${ }^{e}$ Nancy S. Ghanayem, MD, MS, ${ }^{\text {f }}$ and Ronald K. Woods, MD, PhD ${ }^{g}$
}

\section{ABSTRACT}

Objective: Management of chest tubes in adult and pediatric patients is highly variable. There are no published guidelines for pediatric cardiac surgical patients. Our center undertook a quality improvement project aimed at reducing chest tube duration and length of stay in postsurgical pediatric cardiac patients.

Methods: A work group identified 2 opportunities for reducing chest tube duration: standardizing removal criteria and increasing frequency of assessment for removal. An algorithm was created, and chest tube assessments were increased to twice daily. All postsurgical cardiac patients were managed according to the algorithm. Outcome measure reporting was limited to patients age 1 month to 18 years with a biventricular surgical procedure. Outcome measures included chest tube duration, cardiac intensive care unit and hospital length of stay, and cost of hospitalization. Process measure was documentation of chest tube assessments. The balancing measure was chest tube reinsertions.

Results: Between April 2016 and July 2018, 126 patients aged 1 month to 18 years underwent a biventricular surgical procedure. Mean chest tube duration decreased from 61 to 47 hours. Cardiac intensive care unit length of stay decreased from 141 hours to 89 hours, hospital length of stay decreased from 266 to 156 hours, and average hospitalization cost decreased from $\$ 75,881$ to $\$ 48,118$. There was no increase in chest tube reinsertions.

Conclusions: Implementation of a chest tube removal algorithm for pediatric cardiac surgery patients resulted in decreased chest tube duration and was associated with decreased length of stay and costs without an increase in reinsertions. More significant impact may be attainable with more aggressive approach to removal. ( $\mathrm{J}$ Thorac Cardiovasc Surg 2019;158:1209-17)

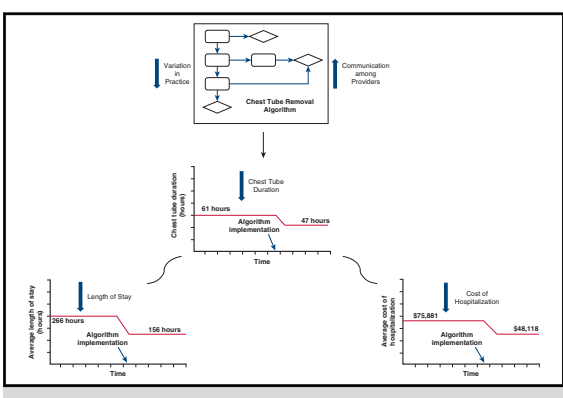

Chest tube removal algorithm reduces CT duration and LOS and costs.

\section{Central Message}

Use of a CT removal algorithm in pediatric patients undergoing cardiac surgery reduced CT duration and was associated with decreased LOS and costs with no increase in reinsertions.

Perspective

There are no published guidelines on management of CTs in pediatric patients undergoing cardiac surgery. Studies have shown that standardizing care reduces LOS and cost. Implementation of a CT removal algorithm at our institution led to a reduction in CT duration and was associated with decreased LOS and costs without an increase in $\mathrm{CT}$ reinsertion rate.

See Commentary on page 1218 .
Increasing health care costs have created an environment of increasing scrutiny of current health care delivery, payment, and reimbursement models. As payment models shift from "pay for volume" to "pay for performance" and with the

\footnotetext{
From the Divisions of ${ }^{\mathrm{a}}$ Critical Care, ${ }^{\mathrm{b}}$ Cardiology, ${ }^{\mathrm{c}}$ Anesthesiology, and ${ }^{\mathrm{g}}$ Cardiothoracic Surgery, Department of Pediatrics, Medical College of Wisconsin, Milwaukee, Wis; 'Herma Heart Institute, Children's Hospital of Wisconsin, Milwaukee, Wis; 'Department of Quality and Patient Safety, Children's Hospital of Wisconsin, Milwaukee, Wis; and ${ }^{\mathrm{f}}$ Division of Critical Care, Department of Pediatrics, Baylor College of Medicine, Houston, Tex.

Institutional Review Board approval waived.

Received for publication Jan 4, 2019; revisions received March 15, 2019; accepted for publication March 30, 2019; available ahead of print May 27, 2019.

Address for reprints: Rebecca A. Bertrandt, MD, Pediatric Critical Care Children's Hospital of Wisconsin, PO Box 1997, Mail Stop 681, 9000 W. Wisconsin Ave, Milwaukee, WI 53226 (E-mail: rarussel@mcw.edu).

$0022-5223 / \$ 36.00$

Copyright (c) 2019 by The American Association for Thoracic Surgery

https://doi.org/10.1016/j.jtcvs.2019.03.120
}

increase in transparency and public reporting, medical leaders, policymakers, and payers face pressure to reduce costs without compromising quality. The Institute for Healthcare Improvement has challenged these groups to achieve the "Triple Aim": improve population health, improve the patient experience, and reduce health care costs. ${ }^{1,2}$ One promising strategy in the quest to realize this Triple Aim is reducing variation in care, which has been

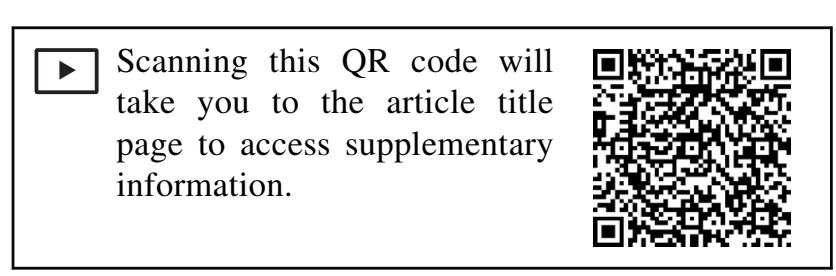




\section{Abbreviations and Acronyms}

$\mathrm{CICU}=$ cardiac intensive care unit

$\mathrm{CT}=$ chest tube

$\mathrm{EHR}=$ electronic health record

PC4 = Pediatric Cardiac Critical Care Consortium

PDSA = plan, do, study, act

QI = quality improvement
LOS $=$ length of stay

Before this project, CT readiness for removal was assessed once daily. To increase the frequency of assessments of CTs for removal, an afternoon assessment of CTs with borderline output in the morning was added (Figure 1).

To track compliance with the new workflow and to gather data on factors influencing CT removal, a tool within the electronic health record (EHR) was created: the "CT rounding navigator." The navigator allowed the providers to record the assessment of each $\mathrm{CT}$ for readiness for removal from a drop-down menu. Drop-down menu selections were chosen on the basis of variables that most often affect the decision to remove or leave a CT in place. CT locations specific to each patient along with 12- and 24-hour volume totals were pulled into the tool directly from the EHR, and the recorded assessments were subsequently transcribed into the patient's daily progress note.

\section{Patient Selection}

All postsurgical patients with CTs managed by the cardiothoracic surgery team were managed according to the algorithm guidelines. Process and balancing measure reporting were applied to this patient population. Subgroup analysis was defined a priori and was performed on a more homogenous cohort to increase the likelihood of detecting change, and outcome measure reporting was limited to this more homogeneous patient group: patients aged 1 month to 18 years with a biventricular surgical procedure. Compliance with documentation of assessments of CTs for readiness for removal was not evaluated on the day of surgery.

\section{Institutional Practices}

CTs placed in patients in the operating room by the cardiothoracic surgery team occupied the mediastinal or pleural space. The number of tubes placed was at the discretion of the surgeon. Technique for placement has not changed. Postoperatively, wall suction ("Pleurovac") was used to manage CTs in most patients. On occasion, bulb suction was used for smaller CTs in neonates or infants. This was also at the discretion of the surgeon.

\section{MATERIALS AND METHODS Institutional Engagement}

In 2015, leadership from Children's Hospital of Wisconsin made a commitment to improve health care delivery while maintaining excellent outcomes. A multidisciplinary QI work group was formed and identified duration of CTs for postoperative cardiac patients as a potential area for improvement. Two main opportunities for reducing $\mathrm{CT}$ duration were identified: standardizing the criteria for removal and increasing the frequency of assessment for removal. A current state assessment was performed to identify unnecessary sources of variation and gaps in communication, which then informed the development of an algorithm for CT removal, including an increased frequency of assessment of CTs for readiness for removal.

\section{Care Standardization}

After completion and analysis of the current state assessment, an algorithm for postoperative chest removal was created (Figure 1). The workflow illustrated the many variables for consideration when assessing readiness for CT removal. These variables were chosen by a group of providers with representation from cardiothoracic surgery, cardiac critical care, cardiology, and nursing. The process of selecting the variables was led by a QI specialist. The volume criteria were based on a preimplementation exploratory data analysis of CT drainage in the 36 hours before removal from 488 CTs associated with 283 admissions in the preceding 1.75 years (Table 1). CTs occupied the mediastinal or pleural space. Volume criteria were conservatively set at 1 standard deviation of the mean for the 12-hour time interval before CT removal for 3 weight ranges (Figure 1). CTs with drainage greater than 3 standard deviations from the mean during any of the 3 time intervals evaluated were excluded from the exploratory analysis.

\section{Measures}

The primary outcome measure was CT duration. Secondary outcome measures included cardiac intensive care unit (CICU) stay, hospital LOS, and hospitalization cost. Hospital costs included indirect costs (eg, billing, legal, and human resources, and others) and direct costs of hospitalization (eg, room, nursing, devices, laboratory services and others) prorated to the day. CT duration (hours) was calculated per patient from the first placement time to the last removal time, regardless of the number of CTs. LOS is reported in hours.

Process measures included compliance with 2 types of assessment: documentation of morning assessment of readiness for removal and afternoon assessment or removal of CTs found to have borderline volume in the morning. Compliance with the morning assessment required documentation by noon and use of the $\mathrm{CT}$ rounding navigator. Percent documentation was calculated weekly on the basis of the number of daily assessments completed and total number of CTs eligible for documentation each day. The second process measure was compliance with performing an afternoon assessment of CTs found to have borderline volume for removal in the morning. The number of afternoon CT removals was also tracked.

$\mathrm{CT}$ reinsertions were tracked as a balancing measure. The Pediatric Cardiac Critical Care Consortium (PC4) database was used to identify patients who had CTs inserted for pleural effusion (excludes CTs placed in the operating room). The PC4 database is a clinical database dedicated to improved quality of care of pediatric patients and patients with congenital heart disease with participation from 52 CICUs across the United States and Canada. ${ }^{17}$ Of those patients identified from local PC4 records, chart review was performed to identify those who met criteria for a CT reinsertion. For the purposes of this QI project, this was defined as reinsertion of a 

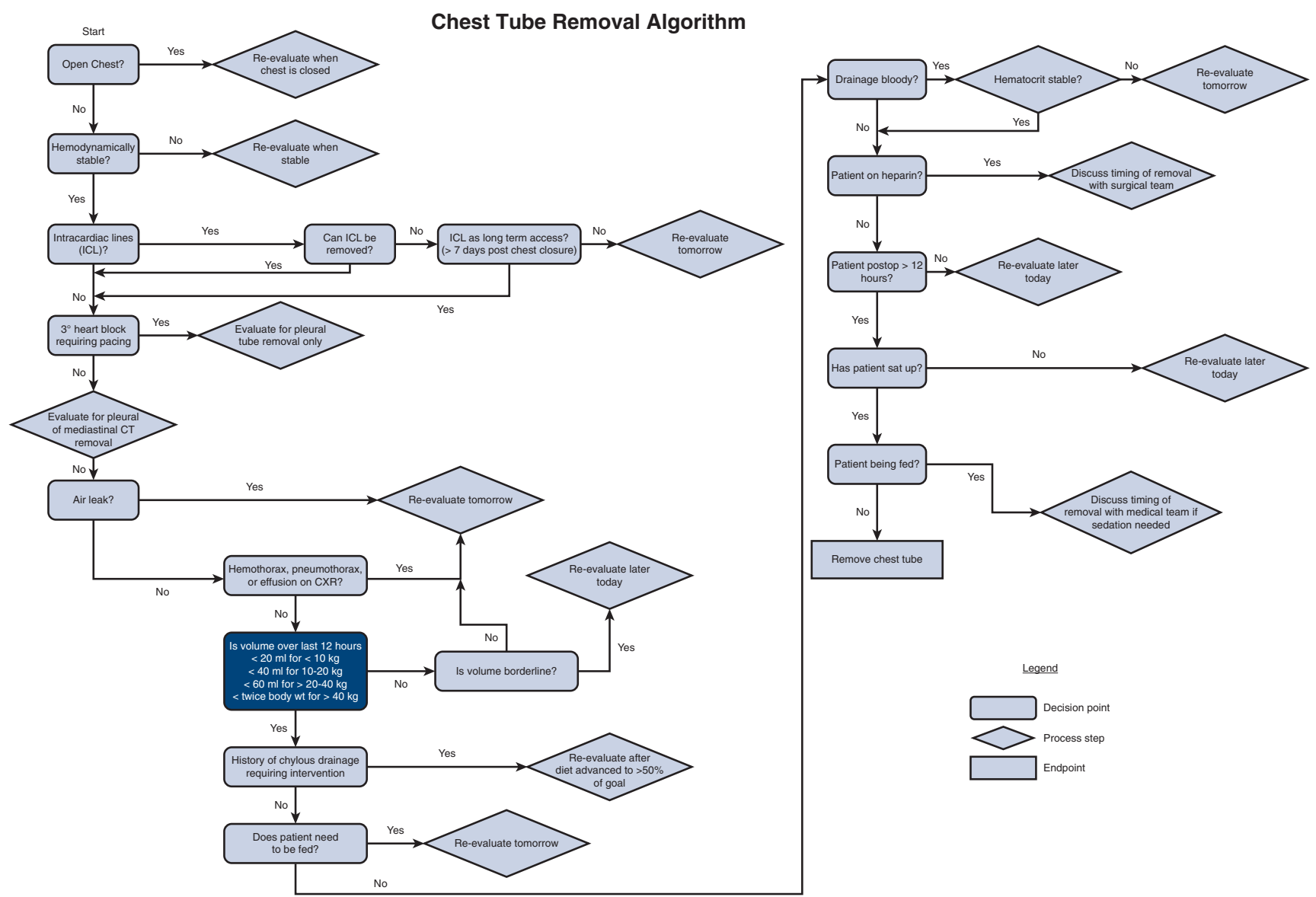

FIGURE 1. CT removal algorithm. $I C L$, Intracardiac line; $C T$, chest tube; $C X R$, chest x-ray.

CT after removal of a tube in the same pleural or mediastinal space within the preceding 7 days. Our institution began contributing to the PC4 database 6 months before algorithm implementation. Therefore, preimplementation reinsertions were readily identified and reported for that time interval.

\section{Control Chart Methodology}

Outcome measures were reported using control charts (Figures 2-5). Baseline mean values were established using the mean of all available data points. A new mean was calculated with each shift, which was defined as 8 consecutive data points above or below the previously established mean. Upper and lower control limits represent 3 standard deviations above and below the mean, respectively.

\section{RESULTS}

Outcome Measures (Postsurgical Patients 1 Month to 18 Years With Biventricular Surgical Procedure)

Between April 2016 and July 2018, 126 patients aged 1 month to 18 years underwent a biventricular surgical

TABLE 1. Average chest tube output (milliliters) before removal by weight (historical data)

\begin{tabular}{|c|c|c|c|c|}
\hline Weight group & N (CTs) & $<12 \mathrm{~h}$ before removal & $12-24 \mathrm{~h}$ before removal & $25-36 \mathrm{~h}$ before removal \\
\hline$<10 \mathrm{~kg}$ & 375 & $\begin{array}{l}9.2 \pm 11.2 \\
\text { median } 7 \\
\mathrm{~N}=375\end{array}$ & $\begin{array}{l}13.3 \pm 14.9 \\
\text { median } 10 \\
\mathrm{~N}=373\end{array}$ & $\begin{array}{l}21.0 \pm 21.6 \\
\text { median } 15 \\
\mathrm{~N}=353\end{array}$ \\
\hline $11-20 \mathrm{~kg}$ & 100 & $\begin{array}{l}15.4 \pm 16.0 \\
\text { median } 10 \\
\mathrm{~N}=100\end{array}$ & $\begin{array}{l}28.9 \pm 24.2 \\
\text { median } 22 \\
\mathrm{~N}=100\end{array}$ & $\begin{array}{l}38.2 \pm 31.3 \\
\text { median } 37 \\
\mathrm{~N}=90\end{array}$ \\
\hline$>20 \mathrm{~kg}$ & 13 & $\begin{array}{l}30.8 \pm 30.7 \\
\text { median } 13 \\
\mathrm{~N}=13\end{array}$ & $\begin{array}{l}39.5 \pm 28.2 \\
\text { median } 36 \\
\mathrm{~N}=13\end{array}$ & $\begin{array}{l}53.4 \pm 46.0 \\
\text { median } 44 \\
\mathrm{~N}=11\end{array}$ \\
\hline
\end{tabular}

Average CT output (mL) for 3 time intervals in patients (age 1 month to 7 years) grouped by weight from 283 admissions with biventricular procedure between January 2013 and September 2015. Data are expressed as mean (standard deviation) and median milliliters. Patients with a volume greater than 3 standard deviations from the mean in any time interval were excluded. $C T$, Chest tube. 


\section{Chest Tube Duration X-bar Chart}

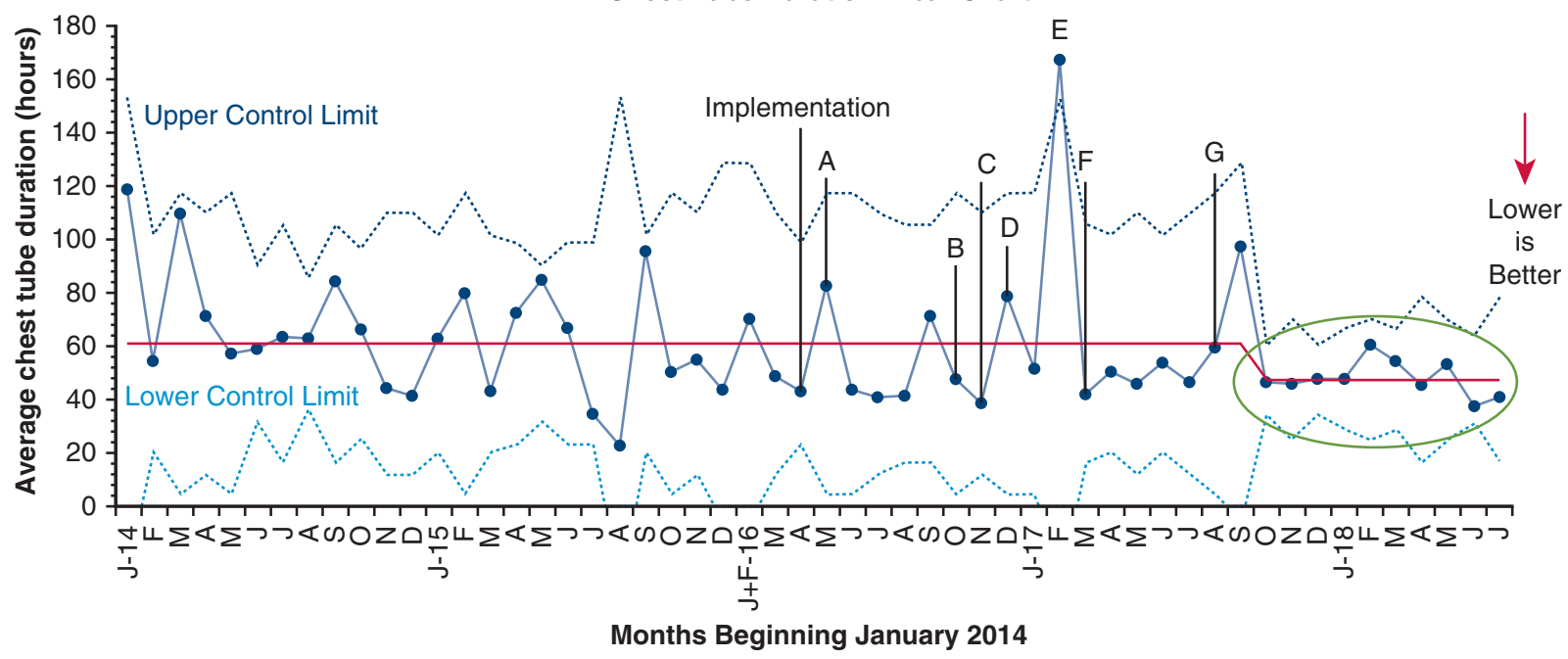

Annotation Key:

Implementation of chest tube (CT) removal algorithm on 4/4/16

A (5/4/16): Modification to CT Rounding Navigator

B (10/15/16): RN education on standardized CT removal documentation

C (11/30/16): Root cause analysis of inconsistent documentation of CT assessments
D (12/29/16): Reinforced workflow with cardiothoracic surgery team E (2/15/17): Modification to CT Rounding Navigator

F (3/20/17 -3/29/17): RN Education Days focused on CT removal process

G (8/21/17): Positive shift in compliance with documentation of CT assessments

FIGURE 2. Average CT duration in hours by month (blue data points) for patients aged 1 month to 18 years with biventricular procedure ( $\mathrm{n}=300,174$ patients preimplementation, 126 patients postimplementation). Duration $=$ placement of first CT to removal of CT per patient. Timing of algorithm implementation noted (April 2016). Red line = mean of the first 26 data points of the preimplementation era (61 hours), decreasing to 47 hours in October 2017. New mean is calculated with each shift ( 8 consecutive data points above/below previous mean), marked with green circle. Hashed lines represent upper and lower control limits ( 3 standard deviations above/below the mean). Letters A to G mark PDSA interventions. $C T$, Chest tube.

procedure and were included in the CT removal algorithm. There were $277 \mathrm{CTs}$ in these 126 patients $(2.2$ CTs per patient on average). Median age of this cohort was 8.3 months (0.37-4.0). Of note, surgical complexity pre- and postimplementation was similar, with average Society of Thoracic Surgeons/European Association for Cardio-Thoracic Surgery mortality score 1.9 preimplementation $(\mathrm{n}=161)$ and 1.7 postimplementation $(\mathrm{n}=124)$ (2 patients with procedures not mapped to Society of Thoracic Surgeons/European Association for Cardio-Thoracic Surgery scores).

Interventions undertaken as part of this plan, do, study, act (PDSA) process improvement are noted as points $\mathrm{A}$ to $\mathrm{F}$ on Figures 2-6. Point A was an update to the rounding navigator allowing for easier documentation of afternoon CT assessments and removals. This modification improved documentation of CT assessments, communication among providers, and the ability to track CT removal. Education specific to nurses to improve accuracy of CT removal documentation was provided at point B. Because of progressive decline in compliance with documentation of morning assessments between points $\mathrm{A}$ and $\mathrm{C}$, a detailed chart review of patients with missing documentation was performed at point $\mathrm{C}$. This review led to the discovery of multiple barriers which, when addressed, led to significant improvement in compliance with documentation. For example, issues with the EHR triggering (or not triggering) the rounding navigator appropriately resulted in apparent noncompliance with documentation. Variability in timing of CT entry and closure within the EHR among nurses was also discovered. The timing was found to affect functionality of the rounding navigator and therefore the ability of the team to document assessments. Finally, several CTs lacking documentation were found to be those placed and managed by services other than cardiothoracic surgery. Systematically eliminating these tubes from the denominator led to improvement in measured compliance with documentation. At point $\mathrm{D}$, documentation workflow was reinforced with the cardiothoracic surgery team, and the rounding navigator was optimized again at point $\mathrm{E}$. This update to the navigator allowed the cardiothoracic surgery team to document their assessments up to 6 hours after CT removal. Investigation of suboptimal compliance with documentation revealed cases where the rounding navigator failed to launch immediately after bedside nurses documented CTs as having been removed. Addition of the 6-hour window improved accuracy of measured compliance with documentation. Nursing Education Day took place in March 2017 (Point F), at which time general education was repeated. Finally, Point G indicates a sustained positive shift in compliance with documentation of CT assessments. 


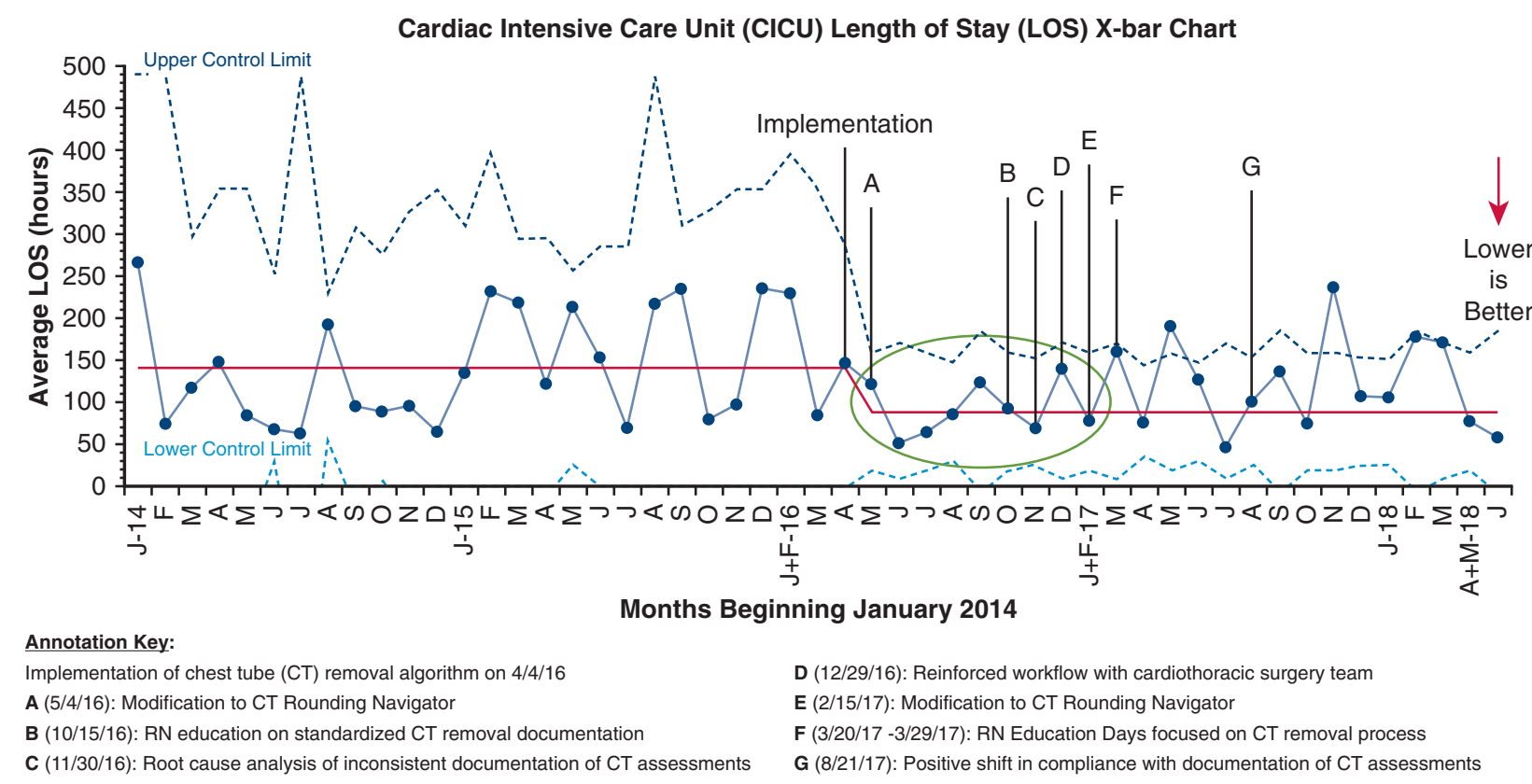

FIGURE 3. Average CICU LOS in hours by month (blue data points) for patients aged 1 month to 18 years with biventricular procedure ( $\mathrm{n}=285$, 159 preimplementation, 126 postimplementation). Patients with CICU LOS more than 1000 hours excluded. Timing of algorithm implementation noted (April 2016). Red line $=$ mean of first 26 data points of the preimplementation era ( 141 hours). The new mean of 89 hours is calculated with the downward shift, indicated by the data points within the green circle. Hashed lines represent upper and lower control limits ( 3 standard deviations above/below the mean). Letters A to G mark PDSA interventions. $C T$, Chest tube.

Average CT duration decreased from 61 hours to 47 hours after implementation of the CT removal algorithm (Figure 2). CT duration was calculated from the time of insertion of the first $\mathrm{CT}$ to the removal of the last $\mathrm{CT}$ for each patient (174 patients preimplementation and 126 patients postimplementation). Two patients with CT duration greater than 330 hours and shown to be clear outliers on a graph of distribution of CT duration (not shown) were excluded. An outlier was apparent at Point E. Upon review, the markedly elevated average CT duration for this month was due in large part to 1 patient with a complicated postoperative course and a CT duration of 287 hours.

Figure 3 shows the average CICU LOS by month from January 2014 to June 2018 for patients aged 1 month to 18 years with a biventricular procedure. Average CICU LOS decreased from 141 hours to 89 hours (5.9 days to 3.7 days). A reduction in average hospital LOS from 266 hours to 156 hours ( 11.1 days to 6.5 days) was also observed (Figure 4). In November 2017, an outlier was apparent. Upon review, the average LOS for this month was increased in large part because of 1 patient with a hospital LOS of 1873 hours (78 days) whose surgery was delayed because of infectious concerns and who struggled postoperatively with feeding.

A convenient sample was used to calculate preimplementation hospital costs. The sample included historical controls used to create volume criteria in initial current state assessment for whom cost data were readily accessible (beginning January 2015, $\mathrm{n}=70$ ). These patients differed in age (age 1 month to 7 years vs age 1 month to 18 years) from the cohort used for LOS reporting. As such, patients used to calculate postimplementation hospital costs $(\mathrm{n}=110)$ match this group in age. Average hospitalization costs decreased from $\$ 75,881$ to $\$ 48,118$ for the matched cohort (Figure 5).

\section{Process Measures (All Postsurgical Patients With Chest Tubes Managed by the Cardiothoracic Surgery Team)}

Compliance with morning documentation of CTs for readiness for removal is shown in Figure 6. The $\mathrm{p}$ chart shows the proportion of CTs every 2 weeks for which an AM assessment was done $(n=6667)$ compared with the total number of CTs for which an AM assessment should have been done $(\mathrm{n}=7778)$. A sustained shift is evident since July 2017 where compliance with documentation of CT assessments improved from a mean of $81.7 \%$ to $92.2 \%$.

Compliance with an afternoon assessment of CTs in those patients found to have borderline CT volume in the morning was also tracked. Over the course of the project, compliance with performing an afternoon assessment was $72 \%$ (not shown). Fifty CTs were removed in the afternoon as a result of the increased frequency of assessment of tubes for readiness for removal. 


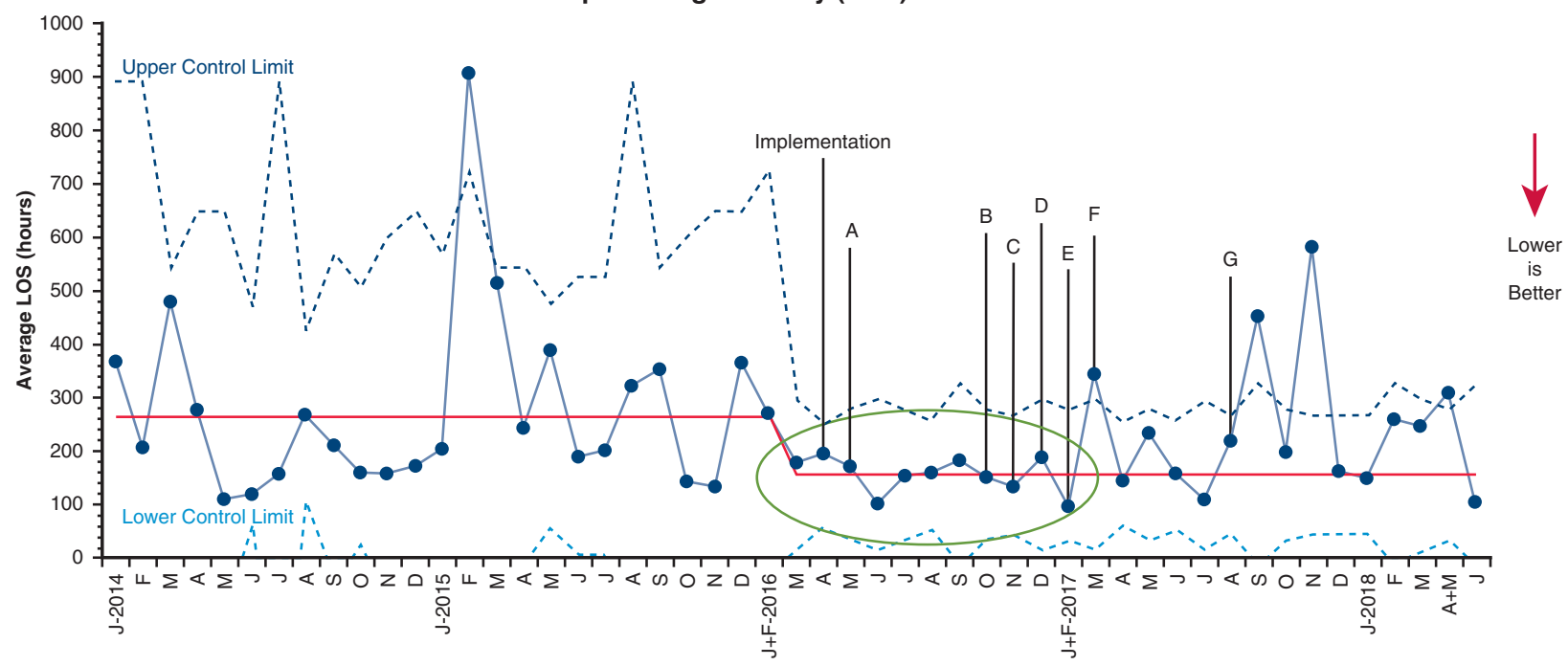

Annotation Key:

Implementation of chest tube (CT) removal algorithm on 4/4/16

A (5/4/16): Improvement of CT Navigator

B (10/15/16): RN education on standardized CT removal documentation

C (11/30/16): Root cause analysis of missing documentation
D (12/29/16): Reinforced workflow with CT surgery team

E (2/15/17): Further improvement of CT Navigator

F (3/20/17 - 3/29/17): RN Education Days focused on CT removal process

G (8/21/17): Positive shift in CT assessment documentation

FIGURE 4. Average hospital LOS in hours by month (blue data points) for patients aged 1 month to 18 years with biventricular procedure (n = 285, 159 preimplementation, 126 postimplementation). Patients with CICU LOS more than 1000 hours excluded. Timing of algorithm implementation noted (April 2016). Red line $=$ mean of first 25 data points of the preimplementation era (266 hours), decreasing to 156 hours in March 2016 . New mean is calculated with each shift ( 8 consecutive data points above/below previous mean), marked with green circle. Hashed lines represent upper and lower control limits ( 3 standard deviations above/below the mean). Letters A to G mark PDSA interventions. CT, Chest tube.

\section{Balancing Measure (All Patients With Chest Tubes Managed by the Cardiothoracic Surgery Team)}

There was no increase in CT reinsertions after implementation of the CT removal algorithm. From the start of the project in April 2016 through July 2018 (26 months), 2 CTs were reinserted, whereas $1 \mathrm{CT}$ was reinserted in the 6 months before project implementation.

\section{DISCUSSION}

Variation in the management of CT removal is multifactorial in nature. Published guidelines on the subject are lacking, and adult literature has focused on patients postlobectomy. To our knowledge, nothing has been published on the management of CTs in the pediatric cardiac surgery population. As such, we developed a QI project aimed at reducing CT duration and LOS for pediatric cardiac surgery patients via 2 main mechanisms: reducing variation in practice and increasing the frequency with which CTs were assessed for readiness for removal. A multidisciplinary team performed a current state assessment to map out decisionmaking related to $\mathrm{CT}$ removal and create a $\mathrm{CT}$ removal algorithm. Rather than relying on anecdotal evidence, historical data were used to guide volume criteria for removal.

Compliance with morning and afternoon assessments of CTs for readiness for removal increased over the course of the project, and the impact of PDSA cycle interventions on both process and outcome measures was evident. Identifying and addressing problems found through the PDSA cycles have resulted in a process that is sustainable. CT reinsertions were rare and not significantly different than the rate before project implementation, suggesting that a more aggressive approach may be appropriate.

The addition of the afternoon assessment received positive responses from nursing staff and families alike. Nurses reported feeling empowered to advocate for their patients, often approaching cardiothoracic surgery team members regarding possible removal of CTs, and patients and families reported improved satisfaction with consideration of earlier removal of tubes, even if only by half a day.

With only 2 CT reinsertions since project implementation and evidence of sustainability, we are poised to achieve greater impact as we move forward with next steps in this QI project. Rare CT reinsertion suggests that a more aggressive approach to removal may be appropriate. For this initial project, drainage 1 standard deviation above the mean for a historic cohort was chosen as a purposefully conservative guideline. Further study could determine whether a larger volume of drainage in the 12- or 24-hour period before removal would result in similarly low reinsertion rate. In addition, a more detailed analysis of data captured in the rounding navigator to better understand factors affecting the decision to remove CTs is under way. 


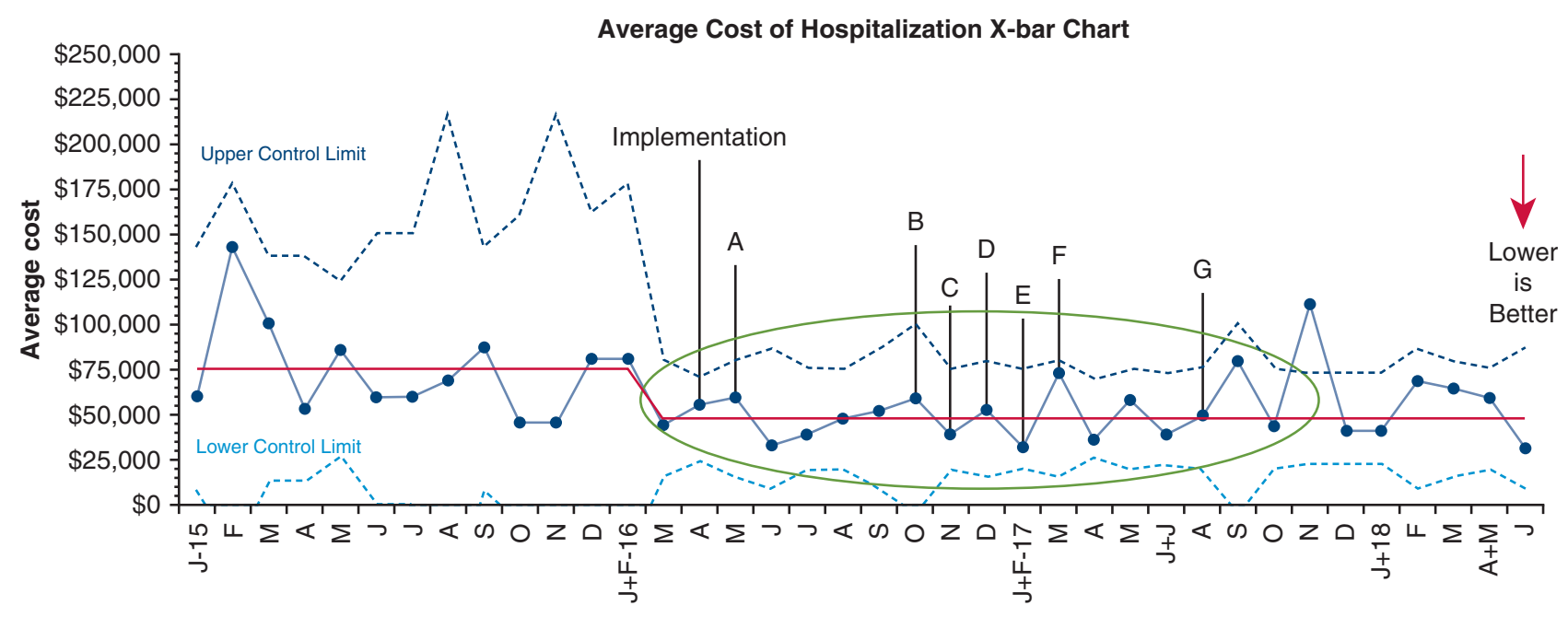

\section{Annotation Key:}

Implementation of chest tube (CT) removal algorithm on 4/4/16

A (5/4/16): Modification to CT Rounding Navigator

B (10/15/16): RN education on standardized CT removal documentation

C (11/30/16): Root cause analysis of inconsistent documentation of CT assessments

D (12/29/16): Reinforced workflow with cardiothoracic surgery team

E (2/15/17): Modification to CT Rounding Navigator

F (3/20/17 -3/29/17): RN Education Days focused on CT removal process

G (8/21/17): Positive shift in compliance with documentation of CT assessments

FIGURE 5. Average cost of hospitalization by month (blue data points) for patients aged 1 month to 7 years with biventricular procedure $(\mathrm{n}=180,110$ preimplementation, 70 postimplementation). Patients with CICU LOS more than 1000 hours excluded. Timing of algorithm implementation noted (April 2016). Red line $=$ mean of first 13 data points of the preimplementation era $(\$ 75,881)$, decreasing to $\$ 48,118$ in March 2016. New mean is calculated with each shift ( 8 consecutive data points above/below previous mean), marked with green circle. Hashed lines represent upper and lower control limits ( 3 standard deviations above/below the mean). Letters A to G mark PDSA interventions. CT, Chest tube.

We showed a $22 \%$ reduction in $\mathrm{CT}$ duration after project implementation. This was associated with reductions in CICU LOS, hospital LOS, and hospitalization costs, a summary of which is depicted in Figure 7. The observed reduction in CT duration was modest in comparison with the observed reduction in LOS. We believe this could be explained in part by the importance of CT removal as it relates to other steps in postoperative recovery. We see CT removal as a pivotal step in the postoperative period because it heralds a time of increased mobility, less frequent use of

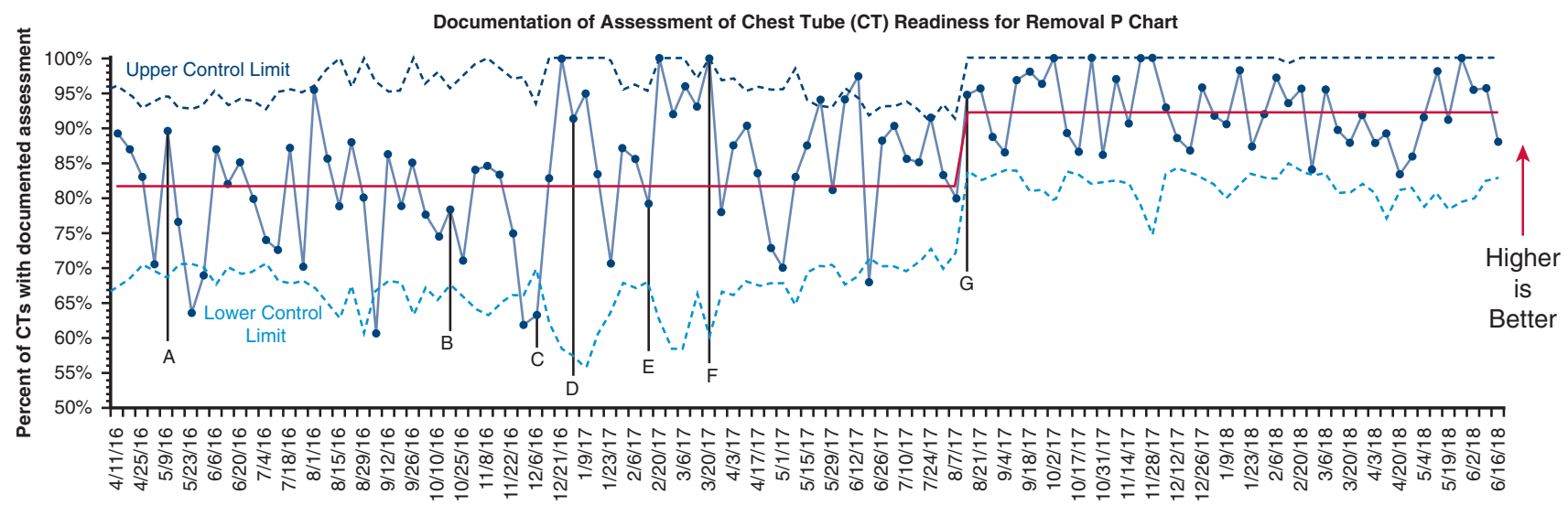

\footnotetext{
Annotation Key:

Implementation of chest tube (CT) removal algorithm on $4 / 4 / 16$ A (5/4/16): Modification to CT Rounding Navigator

B (10/15/16): RN education on standardized CT removal documentation

C (11/30/16): Root cause analysis of inconsistent documentation

of CT assessments
}

$$
\begin{aligned}
& \text { D (12/29/16): Reinforced workflow with cardiothoracic surgery team } \\
& \text { E (2/15/17): Modification to CT Rounding Navigator } \\
& \text { F (3/20/17 -3/29/17): RN Education Days focused on CT removal process } \\
& \text { G (8/21/17): Positive shift in compliance with documentation of CT assessments }
\end{aligned}
$$

FIGURE 6. Percent of CTs with a documented assessment by week (blue data points). Percent = assessments documented/total CT eligible for documentation per day. Total opportunities for documentation $=7778$. Red line $=$ mean of first 20 data points (81.7\%), increasing to $92.2 \%$ in August 2017 . The new mean is calculated from all remaining data points of this sustained upward shift (beginning at Point G). Hashed lines represent upper and lower control limits (3 standard deviations above/below the mean). Letters A to G mark PDSA interventions. CT, Chest tube. 


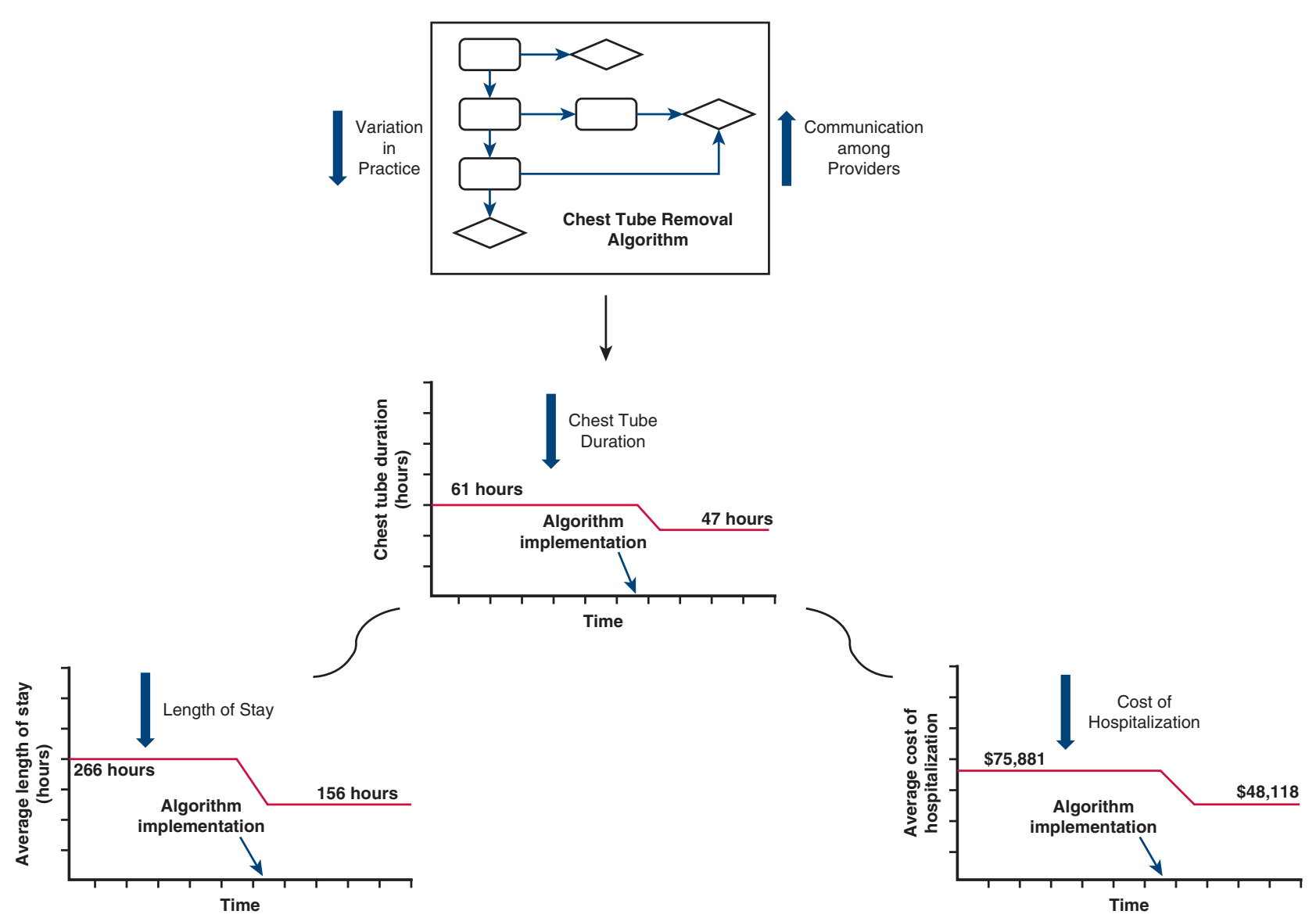

FIGURE 7. ACT removal algorithm was applied to postsurgical patients with CT managed by the cardiothoracic surgery team. Outcome measure reporting was limited to patients aged 1 month to 18 years for CT duration and LOS $(n=126)$ and patients aged 1 month to 7 years for cost of hospitalization $(n=70)$. Average CT duration decreased from 61 to 47 hours. Implementation of the algorithm was associated with a reduction in average hospital LOS from 266 hours to 156 hours, average CICU LOS from 141 hours to 89 hours (not shown), and average hospitalization cost from $\$ 75,881$ to $\$ 48,118$.

narcotics and sedative medications, and a transition to enteral diuretics. However, despite the observed reductions in LOS and cost during the study interval, we caution against over-interpreting the results and inferring proportionate causation. Although we would not necessarily expect a 1-to-1 correlation between reduced hours of CT duration and reduced hours of LOS, the disproportionately larger reduction in LOS and cost argue against CT duration being the sole or dominant explanation for these reductions. It is more likely that reduction in CT duration was one among many improvements occurring at our institution to reduce LOS and cost. Nevertheless, a safe reduction in CT duration is still beneficial to the patient because it reduces discomfort and enables progression of care (Video 1).

\section{Study Limitations}

As discussed, one must be careful about attributing outcomes solely to interventions undertaken as part of the QI project, and we acknowledge that other factors influenced the observed trends. For example, fluid and diuretic management were not controlled. In addition, standardization of CT management and documentation in the EHR likely led to improved communication not only among bedside nurses and those removing the CTs but also among providers involved in discharge planning who may have helped reduce LOS. Additionally, although other qualityimprovement efforts aimed at optimizing care for patients with cardiac disease were in early stages of development at the time of this project, they could have increased providers' overall awareness of ongoing work to improve quality of care and thus contributed to observed trends.

Another limitation is related to the patient population. Although outcome reporting was limited to patients who underwent a biventricular surgical procedure, this group still represents a heterogeneous population with variable congenital and acquired comorbidities that might influence the observed reduction in LOS and costs.

Finally, cost reduction was calculated using total hospital costs per patient and included indirect and direct costs of hospitalization. Depending on the time of discharge, it is 


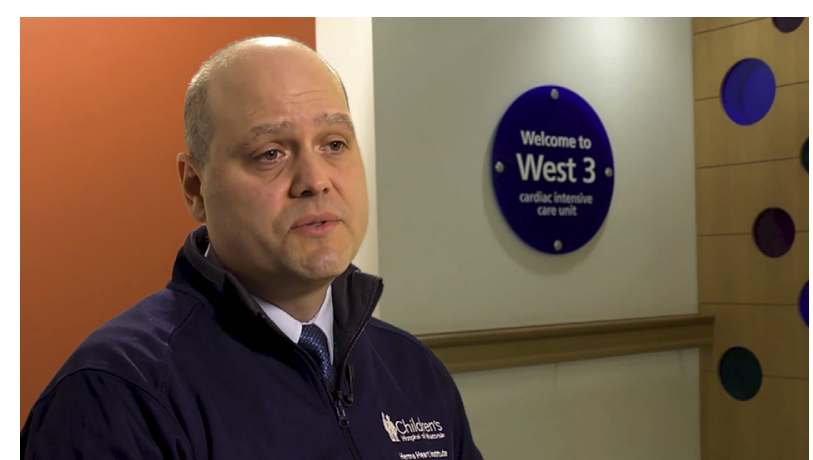

VIDEO 1. After a brief summary of the methods and results of implementing the CT removal algorithm by Becky Bertrandt, MD, Michael Madrzak, PA-C, MPAS, discusses the QI project from the perspective of a member of the cardiothoracic surgery team who participated in this project from start to finish. Video available at: https://www.jtcvs.org/article/S0022-5223(19) 30905-5/fulltext.

possible that cost reduction is overestimated for some encounters if the reduction in LOS did not affect factors that determine cost of hospitalization, such as nursing staffing.

\section{CONCLUSIONS}

Implementation of a CT removal algorithm for postoperative pediatric cardiac surgery patients resulted in decreased CT duration and was associated with reduced LOS and hospitalization costs without leading to an increase in CT reinsertions. The new workflow appears to be sustainable, and more significant reduction in CT duration, LOS, and cost may be attainable with a more aggressive approach to removal.

\section{Conflict of Interest Statement}

Authors have nothing to disclose with regard to commercial support.

The authors thank Lori Plahmer, RN, BSN, MSHSA, and Jessica Schultz for their strong work and contributions to this project.

\section{References}

1. Berwick DM, Nolan TW, Whittington J. The triple aim: care, health, and cost. Health Aff. 2008;27:759-69.

2. Stiefel M, Nolan K. A Guide to Measuring the Triple Aim: Population Health, Experience Of Care, and Per Capita Cost. IHI Innovation Series White Paper.
Cambridge, MA: Institute for Healthcare Improvement; 2012. Available at www.IHI.org. Accessed October 1, 2018.

3. Farias M, Jenkins K, Lock J, Rathod R, Newburger J, Bates D, et al. Standardized clinical assessment and management plans (SCAMPs) provide a better alternative to clinical practice guidelines. Health Aff. 2013;32: 911-20.

4. Rathod RH, Farias M, Friedman KG, Graham D, Fulton DR, Newburger JW, et al. A novel approach to gathering and acting on relevant clinical information: SCAMPs. Congenit Heart Dis. 2010;5:343-53.

5. Muehling B, Schelzig H, Steffen P, Meierhenrich R, Sunder-Plassmann L, Orend $\mathrm{KH}$. A prospective randomized trial comparing traditional and fast-track patient care in elective open infrarenal aneurysm repair. World J Surg. 2009; 33:577-85.

6. Friedman KG, Kane DA, Rathod RH, Renaud A, Farias M, Geggel R, et al. Management of pediatric chest pain using a standardized assessment and management plan. Pediatrics. 2011;128:239-45.

7. Verghese GR, Friedman KG, Rathod RH, Meiri A, Saleeb SF, Graham DA, et al. Resource utilization reduction for evaluation of chest pain in pediatrics using a novel standardized clinical assessment and management plan (SCAMP). J Am Heart Assoc. April 24, 2012 [Epub ahead of print].

8. Porras D, Brown DW, Rathod R, Friedman K, Gauvreau K, Lock JE, et al. Acute outcomes after introduction of a standardized clinical assessment and management plan (SCAMP) for balloon aortic valvuloplasty in congenital aortic stenosis. Congenit Heart Dis. 2014;9:316-25.

9. Vetter TR, Boudreaux AM, Jones KA, Hunter JM Jr, Pittet JF. The perioperative surgical home: how anesthesiology can collaboratively achieve and leverage the triple aim in health care. Anesth Analg. 2014;118:1131-6.

10. Desebbe O, Lanz T, Kain Z, Cannesson M. The perioperative surgical home: an innovative, patient-centred and cost-effective perioperative care model. Anaesth Crit Care Pain Med. 2016;35:59-66.

11. Cerfolio RJ, Bryant AS. Results of a prospective algorithm to remove chest tubes after pulmonary resection with high output. J Thorac Cardiovasc Surg. 2008; 135:269-73

12. Bjerregaard LS, Jensen K, Petersen RH, Hansen HJ. Early chest tube removal after video-assisted thoracic surgery lobectomy with serous fluid production up to 500 ml/day. Eur J Cardiothorac Surg. 2014;45:241-6.

13. Xie HY, Xu K, Tang JX, Bian W, Ma HT, Zhao J, et al. A prospective randomized controlled trial deems a drainage of $300 \mathrm{ml} /$ day safe before removal of the last chest drain after video-assisted thoracoscopic surgery lobectomy. Interact Cardiovasc Thorac Surg. 2015;21:200-5.

14. Zardo P, Busk H, Kutschka I. Chest tube management: state of the art. Curr Opin Anaesthesiol. 2015;28:45-9.

15. Utter GH. The rate of pleural fluid drainage as a criterion for the timing of ches tube removal: theoretical and practical considerations. Ann Thorac Surg. 2013; 96:2262-7.

16. Brunelli A, Beretta E, Cassivi SD, Cerfolio RJ, Detterbeck F, Kiefer T, et al. Consensus definitions to promote an evidence-based approach to management of the pleural space. A collaborative proposal by ESTS, AATS, STS, and GTSC. Eur J Cardiothorac Surg. 2011;40:291-7.

17. Pediatric Cardiac Critical Care Consortium (PC4). Available at: http://pc4 quality.org/. Accessed January 2, 2019.

Key Words: congenital heart disease, perioperative care, quality care, management 